\title{
Estudo fotoelástico do controle vertical com o arco de dupla chave na técnica Straight wire
}

Adriano Dobranszki*, José Henrique Vuolo**, Flamínio Levy Neto***, Hideo Suzuki ${ }^{\star \star \star \star}$,

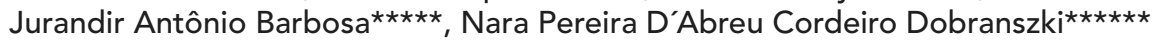

\begin{abstract}
Resumo
Introdução: em alguns casos, a extração de pré-molares torna-se necessária e nem sempre os espaços são completamente fechados após o alinhamento e nivelamento. $\mathrm{O}$ arco de dupla chave, ou Double Key Loop (DKL), é um arco retangular de aço para retração, com duas alças - uma mesial e outra distal ao canino. Objetivos: este trabalho propôs-se a estudar o local onde a força é exercida, após a ativação desse arco, utilizando ativação na alça distal, ativação entre as alças e na alça distal, e ativação com Gurin ${ }^{\circledR}$. Métodos: foram montados nove modelos fotoelásticos de um arco dentário inferior, sem os primeiros pré-molares e os terceiros molares, com braquetes In-Ovation e arco DKL. O arco foi ativado e a região de incisivos, caninos e dentes posteriores foi fotografada, com interposição de filtros polarizadores de luz. Resultados e Conclusões: após a análise do modelo fotoelástico, concluiu-se que a ativação com Gurin ${ }^{\circledR}$ pode produzir movimento de retração anterior com componente extrusivo; a ativação na alça distal pode produzir movimento de retração anterior sem componente extrusivo; e a ativação entre as alças e na alça distal pode produzir movimento de retração anterior com componente intrusivo.
\end{abstract}

Palavras-chave: Fotoelasticidade. Controle vertical. Retração. Arco dupla-chave. DKL. Suzuki's tie.

\section{INTRODUÇÃO}

De maneira geral, existem três razões para a extração dentária em Ortodontia: (a) conseguir espaço para alinhar dentes apinhados, (b) diminuir a protrusão e (c) camuflar problemas moderados de Classes II ou III, quando a correção por modificação do crescimento não é mais possível. Muitas vezes, o espaço conseguido com as extrações não é completamente fechado com o alinhamento dos dentes, sendo necessária uma força adicional para esse fim.
O princípio da fotoelasticidade é baseado no fato de que a maior parte dos materiais claros se torna birrefringente (separação da luz em dois feixes com velocidade e índice de refração diferentes), quando submetidos a estresse mecânico ${ }^{9}$. A birrefringência se manifesta pelo aparecimento de franjas coloridas em áreas de tensão induzida. Em Odontologia, o material reproduz a resiliência e a resistência do periodonto de sustentação ${ }^{6}$, sendo utilizadas cores monocromáticas

* Especialista em Disfunção Temporomandibular e Dor Orofacial (CFO). Mestre em Ortodontia e Ortopedia Facial (Unicastelo / SP). Coordenador do Curso de Especialização em Ortodontia do Instituto de Pesquisa, Ensino e Pós-graduação (IPESP / DF).

** Professor titular de Física da USP - São Paulo / SP.

*** Professor adjunto de Engenharia Mecânica da UnB - Brasília / DF.

**** Mestre em Ortodontia e Ortopedia Facial (Unicastelo / SP). Coordenador do curso de especialização em Ortodontia da São Leopoldo Mandic - Campinas / SP. $\star \star \star \star \star$ Mestre em Ortodontia e Ortopedia Facial (Bauru / SP). Coordenador do curso de especialização em Ortodontia da São Leopoldo Mandic - Campinas / SP.

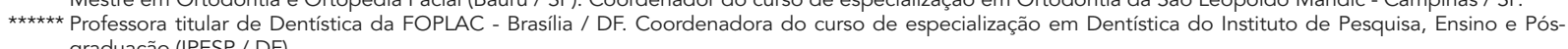
graduação (IPESP / DF). 
para análise da quantidade das forças, enquanto as franjas coloridas fornecem mais informações em relação à direção e distribuição das tensões ${ }^{4}$ (compressão ou tração).

Segundo Roth ${ }^{7}$, o arco com alças duplas tipo buraco de fechadura, DKH ou DKL, poderia ser usado para: (a) permitir ao operador o fechamento completo de espaços com um jogo de arcos; (b) permitir maior controle sobre a inclinação na mecânica de deslizamento; e (c) permitir ao operador eleger como fechar o espaço - com retração anterior, protração posterior e quanto de cada, independente da cooperação do paciente.

O presente estudo se propôs a analisar a direção, distribuição e intensidade da força no ápice radicular de caninos e incisivos, quando o arco DKL é ativado nos modelos fotoelásticos com: (1) ativação na alça distal; (2) ativação entre as alças e na alça distal; e (3) ativação com Gurin ${ }^{\circledR}$.

\section{MATERIAL E MÉTODOS}

O protocolo experimental baseou-se no descrito por Dobranszki ${ }^{3}$, no qual braquetes In-Ovation Roth slot 0,022" (ref. kit 88-055-24, GAC ${ }^{\mathrm{TM}}$, EUA) foram colados com Super-Bonder gel (Loctite $^{\mathrm{TM}}$ ) em protótipos de resina dos incisivos, caninos e segundos pré-molares (Dental Study Model - Nissin Dental Products Inc, Japão - cat. $n^{\circ}$. 091-220). Nos primeiros e segundos molares de resina foram coladas bandas (kit 16-100-21 e 27, GAC ${ }^{\mathrm{TM}}$, EUA) com tubos duplos de slot 0,022" soldados (ref. 69-642-18 e 69-362-18, GAC ${ }^{\mathrm{TM}}$, EUA). O arco DKL (ref. 03-925, GAC ${ }^{\mathrm{TM}}$, EUA) foi inserido nos tubos das bandas e os slots dos demais braquetes foram fechados, de maneira que as raízes ficassem posicionadas como sugerido pela prescrição do aparelho. Foram escolhidos dentes de resina pela grande semelhança anatômica com os dentes naturais, pela sua facilidade de obtenção por meio comercial e para que, se este estudo for reproduzido, haja possibilidade de comparação dos resultados.

Foram colocados $65 \mathrm{ml}$ de água sobre cinco folhas de gelatina transparente Oetker ${ }^{\mathrm{TM}}$, aquecidos em um forno de microondas por 40 segundos na potência máxima. A esse líquido foram adicionados $16 \mathrm{ml}$ de glicerina e homogeneizados por 1 minuto. $\mathrm{O}$ arco com os dentes foi posicionado em um recipiente de acrílico e a gelatina líquida foi vazada sobre o modelo, depois o material foi levado à geladeira por quatro horas, sendo fotografado logo em seguida, sem ser removido do recipiente.

Foram construídos nove padrões fotoelásticos com gelatina, três para cada tipo de ativação. Cada um deles foi fotografado em três posições, em duas tomadas, com e sem o filtro verde de gelatina, para a análise quantitativa e qualitativa: (a) na região dos dentes posteriores (para aferir a calibração da força) com $20^{\circ}$ em relação à objetiva; (b) região dos incisivos com $90^{\circ}$, perpendicular à objetiva; (c) região do canino esquerdo com $130^{\circ}$. Os modelos foram fotografados: (a) com o DKL e ativação na alça distal (Fig. 1); (b) com o DKL e ativação na alça distal e entre as alças (Fig. 2); e (c) com o DKL e ativação com Gurin ${ }^{\circledR}$ (Fig. 3).
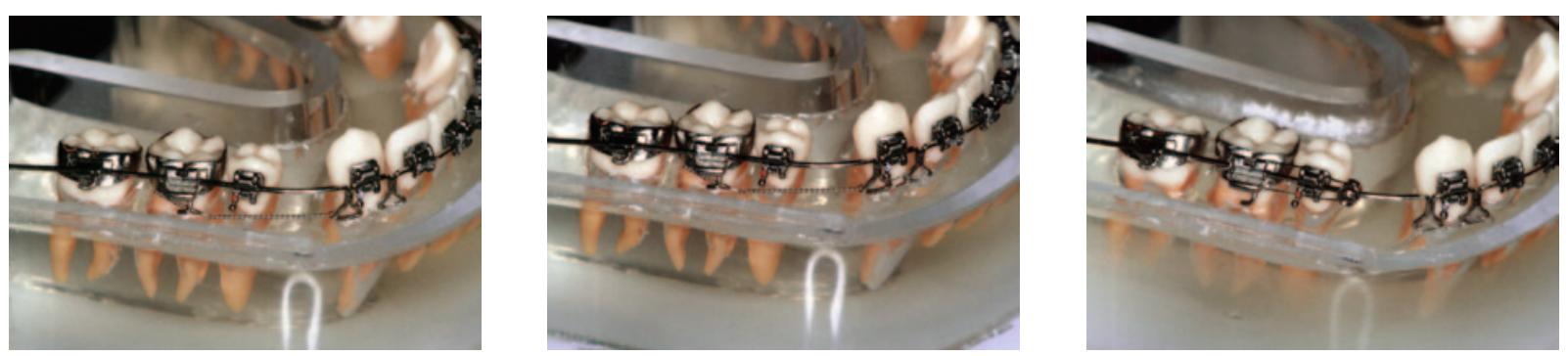

FIGURA 1, 2, 3 - Ativação na alça distal (Suzuki's tie ${ }^{3}$ ), entre as alças e na alça distal, e com Gurin ${ }^{\circledR}$. 
Em todos os modelos, as alças distais do DKL ou os Gurins ${ }^{\circledast}$ foram presos aos ganchos das bandas com amarrilho 0,010", tracionado horizontalmente e torcido até a visualização da primeira franja fotoelástica (amarelo, magenta e ciano) na região dos dentes posteriores.

$\mathrm{Na}$ avaliação qualitativa, as cores foram identificadas próximas à superfície radicular dos incisivos centrais inferiores, nas regiões mesial e distal, e na região apical. Do canino inferior esquerdo, foram observadas as regiões mesial, distal, apical e distoapical da raiz.

Para avaliação quantitativa, a diferença de tons monocromáticos foi delineada, digitalizada e o contorno foi mensurado de forma linear com o programa Corel Draw ${ }^{\circledR}$ 9, com as ferramentas dimensão, dimensão inclinada e dimensão horizontal.

\section{RESULTADOS}

Nesse trabalho, o espectro obtido nos padrões fotoelásticos foi somente até a primeira franja. Antes do aparecimento da franja, a superfície sob estresse adquire um aspecto esbranquiçado, característico da sobreposição de todas as cores. A área do esbranquiçamento aumenta proporcionalmente ao aumento da força aplicada, iniciando com um leve esbranquiçamento ao redor da superfície que transmite a força ao material fotoelástico, até um grande esbranquiçamento, pouco antes do aparecimento da primeira franja. Ultrapassado esse limiar, a sequência de cores das franjas é: primeira franja - amarela, magenta, ciano; segunda franja - amarela, magenta, verde. A figura 4 mostra a região dos quatro incisivos inferiores com o arco DKL e a força resultante da ativação na alça distal, ou Suzuki's tie ${ }^{4}$. A figura 5 mostra o delineamento das áreas de tensão.

As figuras 6 a 9 mostram a região do canino inferior esquerdo com o arco DKL e a força resultante da ativação com Suzuki's tie, Suzuki's tie e ativação entre as alças, a ativação com Gurin ${ }^{\circledR}$ e ativação com Gurin ${ }^{\circledR}$ com interposição do filtro monocromático verde. As setas mostram os vetores de força resultante das ativações.
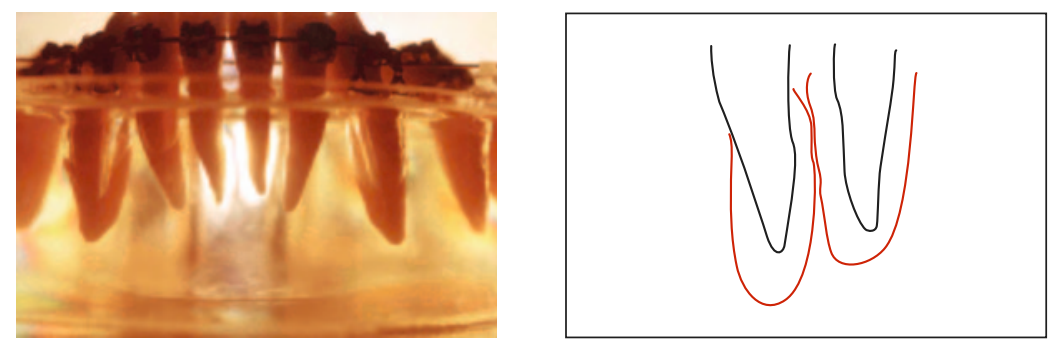

FIGURA 4, 5 - Região de incisivos inferiores com DKL e Suzuki's tie e delineamento da região sob tensão.

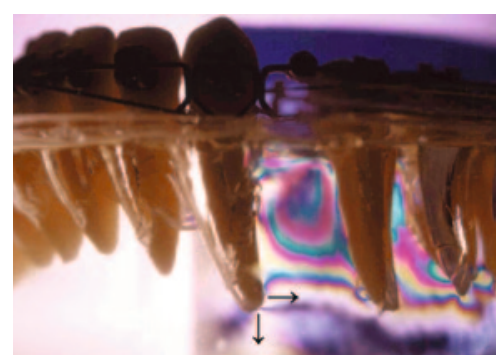

FIGURA 7 - Região de canino com DKL, ativação entre as alças e Suzuki's tie.

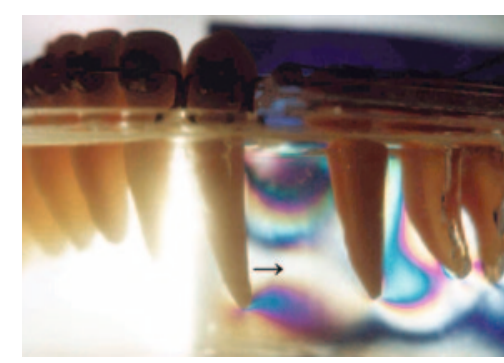

FIGURA 8 - Região de canino com DKL e ativação com Gurin ${ }^{\circledR}$.

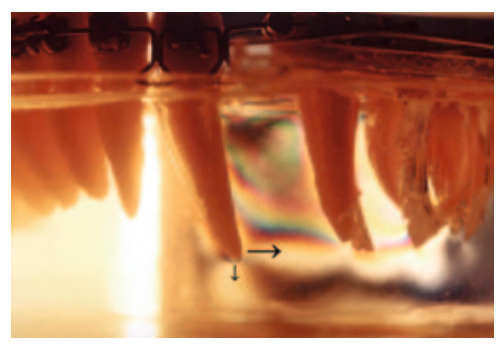

FIGURA 6 - Região de canino com DKL e ativação com Suzuki’s tie.

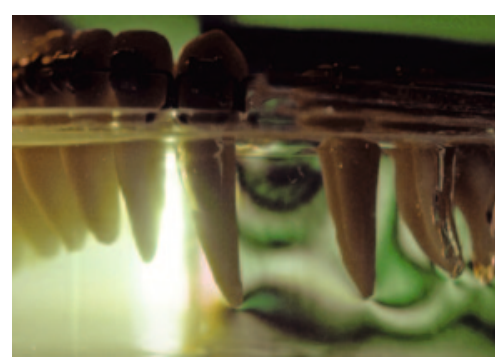

FIGURA 9 - Região de canino com DKL e ativação com Gurin ${ }^{\circledast}$, com interposição de filtro monocromático. 


\section{Avaliação qualitativa dos achados}

A tabela 1 mostra a comparação entre os três tipos de ativação, nas regiões apical, mesial e distal dos incisivos. Os resultados evoluem de ausência de tensões (-) para um pequeno halo esbranquiçado $(+)$, um grande halo esbranquiçado $(++)$, depois para a cor amarela $(+++)$, magenta ou violeta $(++++)$ e, finalmente, ciano ou azul claro $(+++++)$. A avaliação foi feita na região apical das raízes dos incisivos centrais inferiores e na região mesial e distal das raízes dos incisivos centrais inferiores, com resultados idênticos nas três medições em cada tipo de ativação.

\section{Avaliação quantitativa dos achados}

Para todos os padrões fotoelásticos, realizouse a análise quantitativa da extensão das áreas de pressão, a partir dos delineamentos exemplificados na figura 5 (Tab. 3).

\section{DISCUSSÃO}

A razão de incorporar uma dobra gable em um fio é produzir um momento, para que ocorra movimento em corpo do dente ${ }^{2}$. Com a dobra gable, ocorre diminuição no índice de Carga/Deflexão, tornando os níveis de Momento/Força relativamente mais constantes ${ }^{1}$. Na retração dos dentes anteriores, a alça distal do arco de dupla-chave pode ser amarrada na alça mesial ${ }^{8}$, resultando em um efeito semelhante ao gable de $15 \pm 15^{\circ}$. Dessa forma, a força de retração não produziria o movimento indesejado de extrusão dos dentes anteriores.

Normalmente, a correção vertical deve ocorrer antes da anteroposterior ${ }^{5}$, embora seja possível corrigir a sobremordida profunda simultaneamente com a retração ${ }^{8}$, produzindo força intrusiva anterior e extrusiva posterior. Isso pode ser conseguido ativando-se as duas alças com amarrilho e unindo a alça distal à unidade de ancoragem, o que cria um momento para movimento distal de raiz nos dentes anteriores, que tem um componente extrusivo na região do pré-molar, observado, mas não mensurado, no presente trabalho.
A ativação das alças, unindo o Gurin ${ }^{\circledR}$ (distal à segunda alça) à unidade de ancoragem, resultaria em retração com menor força intrusiva no segmento anterior, observado no teste fotoelástico. Teoricamente, o efeito colateral seria de intrusão do segundo pré-molar, observado, mas não mensurado, no modelo com gelatina. $\mathrm{O}$ mesmo efeito pode ser obtido puxando-se o fio e travando-o na distal do segundo molar, ativando as alças como sugerido por Roth ${ }^{7}$.

Na tabela 1, foi feita a comparação dos três tipos de ativação, avaliando a resposta nos incisivos centrais inferiores, tanto nas faces mesiais e distais quanto na região do ápice radicular. Constatouse que, em $100 \%$ das faces mesiais e distais dos incisivos centrais inferiores, ocorreu forte esbranquiçamento, sugerindo que em todos eles existia força de retração.

$\mathrm{Na}$ região apical, observou-se um leve esbranquiçamento na ativação com Gurin ${ }^{\circledR}$, o que pode sugerir uma pequena ou nenhuma força intrusiva. Com a ativação Suzuki's tie, observou-se um grande esbranquiçamento, o que pode sugerir uma moderada força intrusiva. Com a ativação entre as alças e Suzuki's tie, observou-se a formação da primeira franja, com o esbranquiçamento e as cores amarela, magenta e ciano, o que sugere uma grande força intrusiva.

Na tabela 2 foi feita a comparação dos três tipos de ativação, avaliando-se a resposta no canino inferior esquerdo, na face distal e na região do ápice radicular. Constatou-se que, em 100\% das faces distais dos caninos inferiores esquerdos, ocorreu a formação da primeira franja, demonstrando que em todos eles existia força de retração.

Na região apical, observou-se o esbranquiçamento na ativação com Gurin ${ }^{\circledR}$ e, em maior intensidade, com a ativação do Suzuki's tie, sugerindo que no ato da ativação do Suzuki's tie ocorre uma leve força intrusiva. Com a ativação entre as alças e Suzuki's tie, observou-se a formação da primeira franja, com o esbranquiçamento e as cores amarela e magenta, o que sugere uma grande força intrusiva. 
TABELA 1 - Avaliação qualitativa dos incisivos inferiores, idêntica nos 3 modelos.

\begin{tabular}{ccc} 
ativação & $\begin{array}{c}\text { apical } \\
\text { de incisivos }\end{array}$ & $\begin{array}{c}\text { mesial e distal } \\
\text { de incisivos }\end{array}$ \\
\hline $\mathrm{DKL}+$ Gurin $^{\circledR}$ & + & ++ \\
$\mathrm{DKL}+$ Suzuki's tie & ++ & ++ \\
$\mathrm{DKL}+$ Suzuki's tie + alças & ++++ & ++
\end{tabular}

TABELA 2 - Avaliação qualitativa dos caninos inferiores.

$\begin{array}{cccc}\text { ativação } & \begin{array}{c}\text { padrão } \\ \text { fotoelástico }\end{array} & \begin{array}{c}\text { distal do } \\ \text { canino }\end{array} & \begin{array}{c}\text { apical do } \\ \text { canino }\end{array} \\ \text { modelo } 1 & +++++ & + \\ \text { DKL + Gurin } & \text { modelo } 2 & +++++ & + \\ \text { modelo } 3 & ++++ & + \\ \text { DKL + Suzuki's } & \text { modelo } 1 & +++++ & ++ \\ \text { tie } & \text { modelo } 2 & +++++ & ++ \\ \text { modelo } 3 & +++++ & ++ \\ \text { tie }+ \text { alças } & \text { modelo } 1 & +++ & +++ \\ & \text { modelo } 3 & +++++ & ++++ \\ & & +++++ & +++\end{array}$

TABELA 3 - Extensão linear média do contorno das franjas formadas.

\begin{tabular}{cll} 
ativação & incisivos & caninos \\
\hline $\mathrm{DKL}+$ Gurin $^{\circledR}$ & $10,43 \mathrm{~cm}$ & $25,93 \mathrm{~cm}$ \\
\hline $\mathrm{DKL}+$ Suzuki's tie & $12,76 \mathrm{~cm}$ & $23,44 \mathrm{~cm}$ \\
\hline $\mathrm{DKL}+$ Suzuki's tie + alças & $21,29 \mathrm{~cm}$ & $17,79 \mathrm{~cm}$ \\
\hline
\end{tabular}

$\mathrm{Na}$ tabela 3 foi feita a comparação entre os três tipos de ativação, avaliando, de forma aproxima$\mathrm{da}$, a quantidade de tensão produzida pelas ativações do arco. Constatou-se que, na ativação com Gurin $^{\circledR}$, a tensão mecânica foi maior na região do canino, pois produziu um halo com maior perímetro. Na ativação com Suzuki's tie, houve uma distribuição mais uniforme do que na com Gurin ${ }^{\circledR}$ entre os incisivos e canino; na ativação entre as alças e Suzuki's tie, a tensão mecânica foi maior na região dos incisivos, aproximadamente o dobro da obtida com a ativação com Gurin ${ }^{\circledR}$. É importante ponderar que os testes foram feitos com dentes de resina em gelatina, que têm módulo de elasticidade (E) e coeficiente de Poisson ( $v$ ) diferentes do cemento, ligamento periodontal e osso alveolar, por isso sugerimos complementar esses achados qualitativos de forma quantitativa, com outros métodos de avaliação, como, por exemplo, pela Análise de Elementos Finitos.

\section{CONCLUSÕES}

Após a revisão da literatura e o estudo dos resultados obtidos, pode-se concluir que a força aplicada no braquete por meio da ativação do arco DKL produz efeitos na raiz dentária do dentes, da seguinte forma:

\section{Região de incisivos}

- A ativação na alça distal (Suzuki's tie) pode produzir movimento de retração sem intrusão, pois, funcionando como uma dobra gable, produziria uma força contrária à força extrusiva, inerente à contração da alça.

- A ativação entre as alças e Suzuki's tie pode produzir movimento de retração com intrusão e momento (torção) distal das raízes.

- A ativação com Gurin ${ }^{\circledR}$ pode produzir movimento com o menor componente intrusivo entre as diferentes mecânicas testadas, pois não há compensação para o efeito extrusivo inerente à contração da alça do fio de aço, como a dobra gable.

\section{Região de caninos}

- A ativação com o Suzuki's tie pode produzir movimento de retração sem intrusão, pois, funcionando como uma dobra gable, produziria uma força contrária à força extrusiva, inerente à contração da alça.

- A ativação das alças entre si e a ativação com Suzuki's tie podem produzir movimento de retração com intrusão.

- A ativação com Gurin ${ }^{\circledR}$ pode produzir movimento de retração com o menor componente intrusivo entre as diferentes mecânicas testadas, pois não há compensação para o efeito extrusivo inerente à contração da alça, como a dobra gable.

Enviado em: novembro de 2006 Revisado e aceito: maio de 2009 


\title{
Photoelastic study of the vertical control with double key loop archwire in the Straight wire technique
}

\begin{abstract}
Introduction: There are clinical situations in which the extraction of bicuspids becomes necessary and, eventually, the extraction spaces are completely closed after aligning and leveling the teeth. The Double Key Loop (DKL) is a stainless steel arch for retraction, with two loops (keys), one mesial and another distal to the canine. Aim: This study aims to study the area where the force is exerted after the activation of the distal loop, the activation of the loops among themselves and the distal loop, and the activation with Gurin ${ }^{\circledR}$. Methods: Nine photoelastic models of the inferior arch were made, without the first bicuspids and the third molars, with In-Ovation brackets and the DKL. With the interposition of polarizing filters, pictures of incisors canines and posterior teeth were taken, after the activation of the arch. Results and Conclusions: Based on the analysis of the photoelastic model, we concluded that the activation in the distal loop can produce an anterior retraction movement without an extrusive component, while the activation with Gurin ${ }^{\circledR}$ produces an extrusive component; besides that, the activation of the keys among themselves and the distal loop activation can produce an anterior retraction movement with an intrusive component.
\end{abstract}

Keywords: Photoelasticity. Vertical control. Retraction. Double-key archwire. DKL. Suzuki's tie.

\section{REFERÊNCIAS}

1. BURSTONE, C. J.; KOENIG, H. A. Optimizing anterior and canine retraction. Am. J. Orthod. Dentofacial Orthop. St. Louis, v. 70, no. 1, p. 1-19, July 1976.

2. CHACONAS, S. J.; CAPUTO, A. A.; HAYASHI, R. K. Effects of wire size, loop configuration, and gabling on canine-retraction springs. Am. J. Orthod. Dentofacial Orthop., St. Louis, v. 65, no. 1, p. 58-66, Jan. 1974.

3. DOBRANSZKI, A. Estudo fotoelástico com o arco de dupla chave na técnica Straight Wire. 2001. Dissertação (Mestrado)-Universidade Camilo Castelo Branco, Centro de Estudos Odontológicos São Leopoldo Mandic, Campinas, 2001.

4. GLICKMAN, I. et al. Photoelastic analysis of internal stresses in the periodontium created by occlusal forces. J. Periodontol., Chicago, v. 41, no. 1, p. 30-35, 1970.
5. JENNER, J. D. The importance of reducing overbite before overjet with initial treatment mechanics. Aust. Orthod. J., Brisbane, v. 13, no. 4, p. 219-230, Mar. 1995.

6. ROSSATO, C. Estudo fotoelástico das áreas de pressão produzidas no periodonto, por forças ortodônticas, na distalização de canino, pelos métodos convencional e com "power arm". 1982. Dissertação (Mestrado)-Faculdade de Odontologia de Bauru, Universidade de São Paulo, São Paulo, 1982.

7. ROTH, R. H. Treatment mechanics for the straight wire appliance. In: GRABER, T. M.; SWAIN, B. F. Orthodontics: current principles and techniques. St. Louis: C. V. Mosby, 1985. p. 665-716.

8. SUZUKI, H.; LIMA, R. S. Arco de retração anterior dupla chave (DKL - Parker). Ortodontia, São Paulo, v. 34, n. 1, p. 73-78, jan./abr. 2001.

9. VUOLO, J. H. Polarização da luz e displays TN. São Paulo: IFUSP, 1998. p. 1-16.
Endereço para correspondência

Adriano Dobranszki

SRTVS 701 Ed. Centro empresarial Brasília, Bloco A, sala 308

CEP: 70.340-907, Brasília / DF

E-mail: dobranszki@uol.com.br 